\title{
Characterizing the angiogenic activity of patients with single ventricle physiology and aortopulmonary collateral vessels
}

\author{
Nefthi Sandeep, MD, ${ }^{\mathrm{a}, \mathrm{c}}$ Yutaka Uchida, PhD, ${ }^{\mathrm{a}}$ Kanishka Ratnayaka, MD, ${ }^{\mathrm{c}}$ Robert McCarter, $\mathrm{PhD},{ }^{\mathrm{d}}$ \\ Sridhar Hanumanthaiah, MBBS, MS, ${ }^{\mathrm{c}}$ Aminata Bangoura, BS, ${ }^{\mathrm{c}}$ Zhen Zhao, PhD, \\ Jacqueline Oliver-Danna, BS, MT, ${ }^{\mathrm{b}}$ Linda Leatherbury, MD, ${ }^{\mathrm{c}}$ Joshua Kanter, MD, ${ }^{\mathrm{c}}$ and \\ Yoh-suke Mukouyama, $\mathrm{PhD}^{\mathrm{a}}$
}

\begin{abstract}
Objectives: Patients with single ventricle congenital heart disease often form aortopulmonary collateral vessels via an unclear mechanism. To gain insights into the pathogenesis of aortopulmonary collateral vessels, we correlated angiogenic factor levels with in vitro activity and angiographic aortopulmonary collateral assessment and examined whether patients with single ventricle physiology have increased angiogenic factors that can stimulate endothelial cell sprouting in vitro.
\end{abstract}

Methods: In patients with single ventricle physiology $(\mathrm{n}=27)$ and biventricular acyanotic control patients $(\mathrm{n}=21)$, hypoxia-inducible angiogenic factor levels were measured in femoral venous and arterial plasma at cardiac catheterization. To assess plasma angiogenic activity, we used a 3-dimensional in vitro cell sprouting assay that recapitulates angiogenic sprouting. Aortopulmonary collateral angiograms were graded using a 4-point scale.

Results: Compared with controls, patients with single ventricle physiology had increased vascular endothelial growth factor (artery: $58.7 \pm 1.2 \mathrm{pg} / \mathrm{mL}$ vs $35.3 \pm 1.1 \mathrm{pg} / \mathrm{mL}, P<.01$; vein: $34.8 \pm 1.1 \mathrm{pg} / \mathrm{mL}$ vs $21 \pm 1.2 \mathrm{pg} / \mathrm{mL}$, $P<.03$ ), stromal-derived factor 1-alpha (artery: $1901.6 \pm 1.1 \mathrm{pg} / \mathrm{mL}$ vs $1542.6 \pm 1.1 \mathrm{pg} / \mathrm{mL}, P<.03$; vein: $2092.8 \mathrm{pg} / \mathrm{mL} \pm 1.1$ vs $1752.9 \pm 1.1$ $\mathrm{pg} / \mathrm{mL}, P<.02)$, and increased arterial soluble fms-like tyrosine kinase-1, a regulatory vascular endothelial growth factor receptor $(612.3 \pm 1.2 \mathrm{pg} / \mathrm{mL}$ vs $243.1 \pm 1.2 \mathrm{pg} / \mathrm{mL}, P<.003)$. Plasma factors and sprout formation correlated poorly with aortopulmonary collateral severity.

Conclusions: We are the first to correlate plasma angiogenic factor levels with angiography and in vitro angiogenic activity in patients with single ventricle disease with aortopulmonary collaterals. Patients with single ventricle disease have increased stromal-derived factor 1-alpha and soluble fms-like tyrosine kinase-1, and their roles in aortopulmonary collateral formation require further investigation. Plasma factors and angiogenic activity correlate poorly with aortopulmonary collateral severity in patients with single ventricles, suggesting complex mechanisms of angiogenesis. ( $J$ Thorac Cardiovasc Surg 2016;151:1126-35)

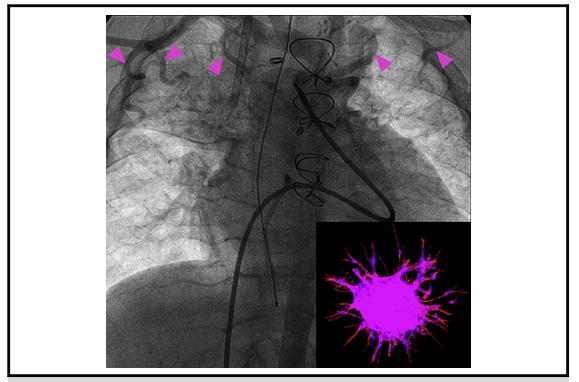

SV patient with Glenn physiology and APCs who had increased angiogenic activity in vitro.

\section{Central Message}

Angiogenic activity of patients with SV physiology correlates poorly with severity of APCs seen angiographically.

\section{Perspective}

Our results suggest that patients with SV physiology with APC vessels exhibit both angiogenically active and angiogenically dormant states. Because it is unclear when and why these patients transition from one state to the other, it will be challenging to develop blood-based biomarker tests with adequate sensitivity, as well as novel and effective medical therapies.

See Editorial Commentary page 1135 .
From the ${ }^{a}$ Laboratory of Stem Cell and Neurovascular Biology, Genetics and Developmental Biology Center, National Heart, Lung, and Blood Institute, National Institutes of Health, Bethesda, Md; ${ }^{\mathrm{b}}$ Department of Laboratory Medicine, National Heart, Lung, and Blood Institute, National Institutes of Health, Bethesda, Md; 'Division of Pediatric Cardiology, Children's National Health System, Washington, DC, and d Department of Biostatistics \& Informatics, Children's National Health System, Washington, DC.

N.S was supported by an institutional Ruth L. Kirschstein National Research Service Award (T32) and National Center for Advancing Translational Sciences (UL1TR000075). This work was supported by the Intramural Research Program of the National Heart, Lung, and Blood Institute, National Institutes of Health (HL006116-05 [Y.-s.M.]). Its contents are solely the responsibility of the authors and do not necessarily represent the official views of the National Center for Advancing Translational Sciences or the National Institutes of Health.

Received for publication June 22, 2015; revisions received Sept 11, 2015; accepted for publication Oct 1, 2015; available ahead of print Nov 21, 2015.

Address for reprints: Yoh-suke Mukouyama, PhD, Laboratory of Stem Cell and Neurovascular Biology, Genetics and Developmental Biology Center, National Heart, Lung, and Blood Institute, National Institutes of Health, Building 10, Room 6C103, MSC1583, 10 Center Drive, Bethesda, Md 20892 (E-mail: mukoyamay@mail.nih.gov). 0022-5223/\$0.00

Published by Elsevier Inc. on behalf of The American Association for Thoracic Surgery

http://dx.doi.org/10.1016/j.jtcvs.2015.10.001 


\section{Abbreviations and Acronyms \\ $\mathrm{APC}=$ aortopulmonary collateral \\ $\mathrm{bFGF}=$ basic fibroblastic growth factor \\ ELISA = enzyme-linked immunosorbent assay \\ HUVEC $=$ human umbilical vein endothelial cell \\ MRI = magnetic resonance imaging \\ SDF-1a $=$ stromal-derived factor 1-alpha \\ sFlt-1 = soluble fms-like tyrosine kinase-1 \\ $\mathrm{SV}=$ single ventricle \\ VEGF $=$ vascular endothelial growth factor}

\section{Supplemental material is available online.}

Single ventricle (SV) heart disease is a broad category encompassing heterogeneous anatomy with an incidence of $0.005 \%$ to $0.01 \%$ in the general population. ${ }^{1,2}$ As these patients undergo palliative 2- or 3-stage surgery in early life, aortopulmonary collateral (APC) blood vessel connections are frequently identified during preoperative cardiac catheterization with an estimated prevalence of $46 \%$ to $71 \%$ after stage 2 palliation and $20 \%$ to $30 \%$ after stage 3 palliation. ${ }^{3-6}$

Our current understanding of how and why APCs form is limited by conflicting data. APCs are suspected to form in part because of hypoxia-inducible angiogenic factors, such as vascular endothelial growth factor (VEGF). ${ }^{7-9}$ Prior studies documented elevated VEGF levels in children with cyanotic heart disease and patients with SV physiology with APCs. ${ }^{8,10}$ A separate study found that serum from cyanotic patients could promote primitive endothelial tube formation in vitro via VEGF signaling. ${ }^{9}$ However, other studies found no relation between VEGF and the presence or severity of APCs. ${ }^{11,12}$ Given our incomplete understanding of why APCs form, we currently rely on palliative therapies such as surgical ligation or catheter-based embolization with metal coils or polyvinyl alcohol particles. However, these are downstream solutions that do not target the primary pathogenic pathway or reliably reduce systemic to pulmonary shunting. ${ }^{3}$

APCs traditionally have been identified via invasive angiography, which requires nontrivial amounts of radiation. Because collateral burden is difficult to quantify by angiography, qualitative subjective grading systems are primarily used. ${ }^{3,13,14}$ Phase-contrast magnetic resonance imaging (MRI) is a noninvasive radiation-free method that can quantify systemic to pulmonary collateral blood flow. ${ }^{15,16}$ However, MRI often requires sedation and is limited by previously implanted hardware (eg, coils) that introduces imaging artifact or is not MRI safe, ${ }^{15}$ although platinum devices can be used to avoid artifact and stainless-steel devices (typically coils) are MRI-safe once endothelialized.

In this study, we hypothesized that functional angiogenic activity of plasma from patients with SV physiology with APCs (SV-APC) would correlate with disease severity. This would make it feasible to develop a blood-based angiogenesis assay to quantify collateral burden without radiation or sedation. More importantly, we might gain mechanistic insights into how APCs form to develop more effective therapies. Because endothelial sprouting is an initial step in forming an immature capillary network, we suspected that APCs may form through a similar pathologic mechanism, and we sought to determine whether plasma in patients with SV physiology with APCs can stimulate cell sprouting in vitro. To this end, we used a modified 3-dimensional in vitro endothelial cell sprouting assay that recapitulates angiogenic sprouting. We used this assay to characterize the angiogenic activity of patients with SV physiology with APCs and hypothesized that patients with greater APC severity would exhibit greater cell sprouting in vitro.

To identify potential causal factors for the diagnosis and treatment of APCs, we also assayed the following factors that are known experimentally and clinically to be involved in sprouting angiogenesis to correlate with our cell sprouting data: VEGF (isoforms A, C, and D), stromal-derived factor 1-alpha (SDF-1a, also known as "CXCL12"), basic fibroblast growth factor (bFGF), and soluble fms-like tyrosine kinase-1 (sFlt-1 or VEGF receptor 1). , $^{8,9,17-24}$

\section{MATERIALS AND METHODS \\ Study Patients}

All patients undergoing aortography during elective cardiac catheterization at Children's National Health System were eligible for enrollment. All patients were approached for enrollment just before their procedure and provided consent for intraprocedural blood collection. We excluded patients with conditions that could confound plasma factor level measurements, such as disorders of hemostasis, malignancy, proteinlosing enteropathy, and active infection or inflammation (eg, myocarditis or Kawasaki's disease). We also excluded patients with central lines because they require heparin that affects angiogenic factor levels. ${ }^{10}$ Finally, we excluded patients with tetralogy of Fallot, pulmonary atresia, and major APC arteries, because these collateral arteries (present in utero) may reflect a different disease process. ${ }^{25}$ Patients were enrolled from March 2014 to March 2015. This study was approved by the institutional review board of Children's National Health System (Washington, DC) and the Institutional Biosafety Committee of the National Heart, Lung, and Blood Institute of the National Institutes of Health (Bethesda, MD).

\section{Aortic Angiography}

Two blinded interventionists (JPK, KR) independently graded all aortic angiograms using the 4-point scale described by Spicer and colleagues ${ }^{13}$ (Figure 1, $A$ ). Grade 1 and 2 collateral vessels did not opacify pulmonary arteries or veins, whereas grade 3 and 4 vessels caused pulmonary vasculature opacification. Grade 1 described a few (ie, $\leq 3$ ) collateral vessels that were small $(<1 \mathrm{~mm})$. Both grade 2 and grade 3 described multiple 
Assessment of aortopulmonary collateral vessel severity

\begin{tabular}{|c|cc|cc|}
\hline Pulmonary opacification & Few & Multiple & Few & Large \\
\hline No & Grade 1 & Grade 2 & Grade 2 & \\
\hline Yes & & Grade 3 & Grade 3 & Grade 4 \\
\hline
\end{tabular}

A

Few: $\leq 3$ vessels, Small: $<1 \mathrm{~mm}$

VEGF-A vs. APC grade

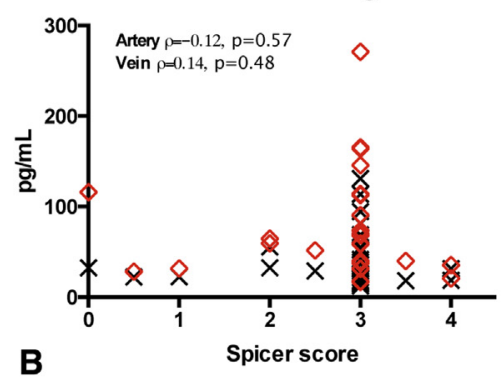

SDF-1a vs. APC grade

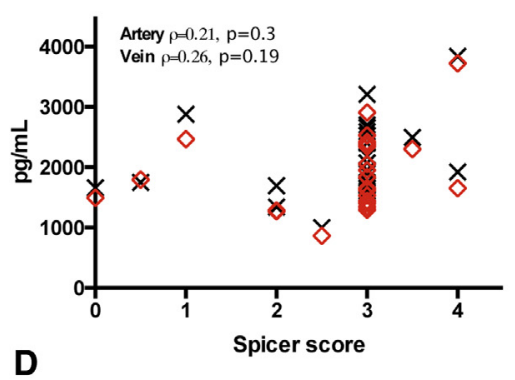

sFIt-1 vs. APC grade

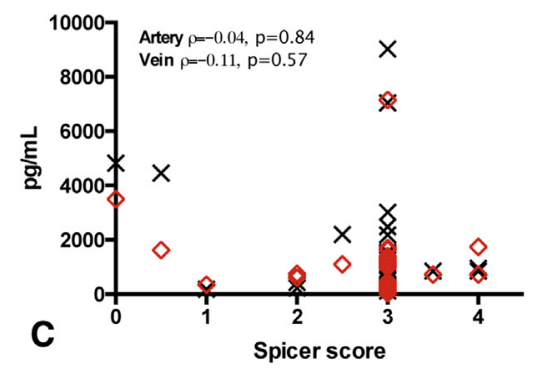

$\diamond$ Artery

$\times$ Vein

Cell sprouts vs. APC grade

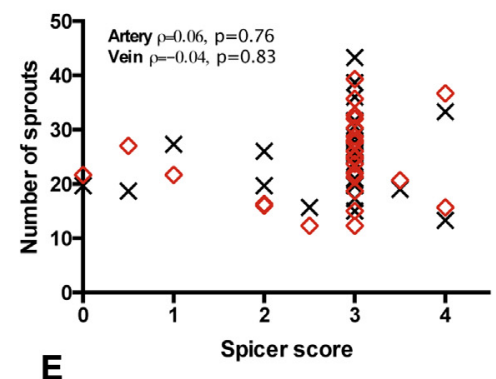

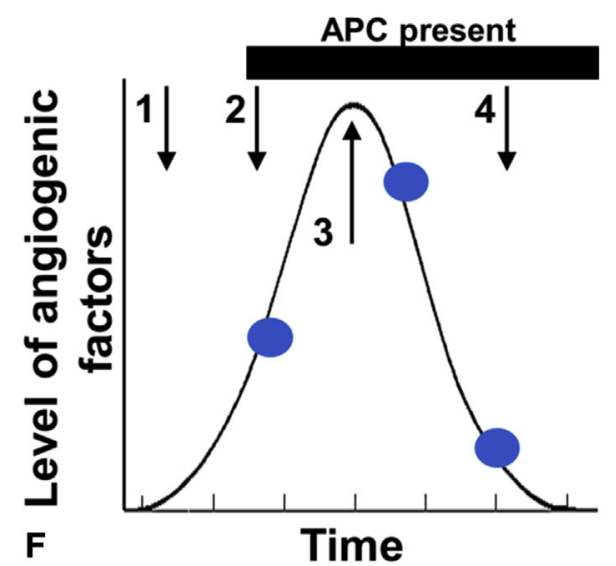

FIGURE 1. Levels of plasma angiogenic factors sampled from the femoral artery (red diamond) and vein (black cross), and sprouting activity in all 27 patients with SV-APC were correlated with APC severity (ie, Spicer score). Spearman's rho ( $\rho$ ) and $P$ values are shown for each graph. A, Spicer scoring criteria. B, Plasma VEGF-A levels. C, Plasma sFlt-1 levels. D, Plasma SDF-1a levels. E, Cell sprouting activity. F, A proposed mechanism describing a possible time-dependent relationship between angiogenic factor levels and APC formation. The black bar represents when APCs can be visualized with contrast angiography. At time 1, a stimulus for APC formation triggers an upregulation of angiogenic factors. At time 2, APCs are angiographically visible while factors continue to upregulate. At time 3, angiogenic factors have reached their peak level and the initial stimulus for APC formation is extinguished. By time 4, factors have downregulated back to normal levels. Blue dots represent possible time points when various patients with SV-APC may have been enrolled, when APCs are visible but factor levels can vary widely. VEGF, Vascular endothelial growth factor; APC, aortopulmonary collateral; $s F l t-1$, soluble fms-like tyrosine kinase 1; $S D F-1 a$, stromal-derived factor. 
(ie, >3) collateral vessels that were small or few vessels that were large. Grade 4 severity described multiple large vessels. Inter-rater reliability was calculated and scores were averaged when there was disagreement. After study enrollment and blood collection, 3 patients (2 patients with SVAPC and 1 control) had left ventricular angiograms instead of aortic angiograms.

\section{Blood Collection and Analysis}

Blood was drawn from the femoral artery and vein after placement of access sheaths and before heparin administration because of its effect on factor levels. ${ }^{10}$ A small amount of venous blood was used for complete blood count with differential analysis. The remaining venous and arterial blood was aliquoted into K3 EDTA Vacutainers for plasma isolation (BD, Franklin Lakes, NJ). All blood was centrifuged within 1 hour of collection at 1100 to $1300 \mathrm{~g}$ for approximately 15 minutes. Additional centrifugation at $10,000 \mathrm{~g}$ for 10 minutes was performed for all SDF-1a plasma to remove platelet fragments per manufacturer instructions. All isolated plasma was stored immediately at $-80^{\circ} \mathrm{C}$ for later use in enzyme-linked immunosorbent assays (ELISAs) and cell sprouting experiments. Total protein analysis was performed on leftover plasma from ELISA and sprouting experiments to ensure similar levels between both patient groups.

\section{Measurement of Plasma Angiogenic Factors}

Colorimetric ELISA kits were used for SDF-1a (R\&D Systems, Minneapolis, Minn), and multi-analyte electrochemoluminescence ELISA kits were used for VEGF-A, C, and D, sFlt-1, and bFGF (MesoScale Discovery, Rockville, Md). All assays were run according to the manufacturer's instructions. All plasma samples were assayed in duplicate and averaged. The lower limits of quantification were $47 \mathrm{pg} / \mathrm{mL}$ for SDF-1a, $5 \mathrm{pg} / \mathrm{mL}$ for VEGF-A, $146 \mathrm{pg} / \mathrm{mL}$ for VEGF-C, $67 \mathrm{pg} / \mathrm{mL}$ for VEGF-D, $10 \mathrm{pg} / \mathrm{mL}$ for sFlt-1, and $3 \mathrm{pg} / \mathrm{mL}$ for bFGF.

\section{Cell Sprouting Assay}

We modified a previously reported 3-dimensional sprouting assay to develop an assay using human umbilical vein endothelial cells (HUVEC) in a collagen matrix. ${ }^{26}$ Complete details are available in the Appendix. In summary, commercially available pooled donor HUVECs were cultured into macrospheres of approximately 2000 cells, placed into a 3-dimensional collagen matrix, and incubated in the presence of $1 \mu \mathrm{L}$ of venous or arterial patient plasma diluted in $2 \mathrm{~mL}$ of serum-free and factor-free endothelial cell basal media at $37^{\circ} \mathrm{C}$ for 20 to 21 hours. Spheres were fixed with $4 \%$ paraformaldehyde, stained with fluorescent dyes (Topro3, Phalloidin), and visualized using a confocal microscope. The number of induced sprouts was counted manually. Each sample was tested in triplicate and averaged.

\section{Statistical Analysis}

All continuous data (eg, number of sprouts, biomarker [factor] levels) are summarized as mean values with $95 \%$ confidence intervals and were analyzed using the unpaired Student $t$ test after log-transformation to meet the normality assumption. Age was analyzed using Wilcoxon's rank-sum test, and all values represent age-adjusted estimates with their corresponding $P$ values. The magnitude and statistical significance of association were evaluated using repeated-measures linear regression for continuous parametric data and Spearman's rank correlation analysis for nonparametric data. Inter-rater reliability in scoring angiograms was calculated using a kappa statistic. By using the significance level of .05 , the study sample size provided $80 \%$ power to detect an effect size difference of 0.7 standard deviation for the number of sprouts formed and 0.8 standard deviation for ELISA measurements. All data were analyzed using Stata 13 software (StataCorp LP, College Station, Tex).

\section{RESULTS \\ Demographics}

Fifty-four consecutively consenting patients were enrolled, and 6 patients were excluded: Two patients did not undergo aortograms after blood collection, 2 patients with SV-APC were acyanotic, 1 patient with SV-APC underwent orthotopic heart transplantation less than 1 year before enrollment, and 1 patient had abnormally low total protein. Of the remaining patients, 21 were acyanotic controls with biventricular circulation and 27 had SV physiology. Summary and individual demographic data are provided in Tables 1 and E1. Miscellaneous control group diagnoses included mitral stenosis/regurgitation, d-transposition of the great arteries after the arterial switch

TABLE 1. Demographic data

\begin{tabular}{lcc}
\hline & Control & SV physiology \\
\hline $\begin{array}{l}\text { Summary data } \\
\mathrm{n}(\%)\end{array}$ & $21(44 \%)$ & $27(56 \%)$ \\
$\quad \begin{array}{l}\text { No. of male patients, } \\
\quad \text { No. of female patients }\end{array}$ & 12,9 & 15,12 \\
$\quad \begin{array}{l}\text { Median catheterization } \\
\text { age, mo (range) }\end{array}$ & $83.5(3-237)$ & $27.5(3-183) *$ \\
Median weight, & & \\
$\quad$ kg (range) & $20(3.8-120.7)$ & $12.4(5.8-67.8)$
\end{tabular}

Diagnosis (n)

$\begin{array}{ll}\text { Valvar or subvalvar } & \text { DORV (7) } \\ \text { AS (4) } & \\ \text { Repaired coarctation (3) } & \text { HLHS (7) } \\ \text { PDA (3) } & \text { DILV (5) } \\ \text { Transplant, 1V (3) } & \text { Tricuspid atresia/ } \\ & \text { stenosis (4) } \\ \text { Transplant, 2V (2) } & \text { PA/IVS (2) } \\ \text { Transitional AV } & \text { Miscellaneous (2) }\end{array}$

canal (2)

Miscellaneous (4)

Glenn circulation

Fontan circulation

(4)

Norwood with

Sano/mBTS (2)

Pulmonary artery

band (1)

RF perforation of

PV (1)

Mean femoral arterial

$98(98,93-99)$

$83(84,64-92)$ saturation \%

(median, range)

$S V$, Single ventricle; $A S$, aortic stenosis; $D O R V$, double outlet right ventricle; $H L H S$ hypoplastic left heart syndrome; $P D A$, patent ductus arteriosus; $D I L V$, double inlet left ventricle; $I V$, transplant recipient was previously univentricular; $2 V$, transplant recipient was previously biventricular; $P A / I V S$, pulmonary atresia with intact ventricular septum; $A V$, atrioventricular; $m B T S$, modified Blalock-Taussig shunt; $R F$, radiofrequency; $P V$, pulmonary valve. ${ }^{*} P=.03$ (rank-sum test). 
operation, repaired truncus arteriosus, and aortic atresia status after the Yasui and Rastelli procedures. Miscellaneous SV group diagnoses included atrioventricular discordance with L-looped ventricles and pulmonary atresia, and heterotaxy with unbalanced atrioventricular canal.

\section{Angiographic Assessment of Aortopulmonary Collateral Severity}

Among the 48 graded angiograms, disagreement occurred in 4 patients with SV-APC and 2 controls (12.5 $\%$ ), producing a high inter-rater reliability (unweighted $\kappa=0.81$, weighted $\kappa=0.89$ ) (Table E1). In 4 cases (3 with SV-APC, 1 control), the Spicer scores differed by 1 , and in 2 cases ( 1 with SV-APC, 1 control), the scores differed by 2 . In the SV-APC group, 18 of 27 patients $(66.7 \%)$ were rated as grade 3, 2 patients were rated as grade 4, 2 patients were rated as grade 2,1 patient was rated as grade 1, and 1 patient had no observable collaterals.
Because of averaging, 3 patients were classified as grades 0.5, 2.5, and 3.5. Among controls, 3 patients had collaterals with average grades of $1,1.5$, and 2 (Table E1). The presence of a patent ductus arteriosus $(\mathrm{n}=3)$ or a modified Blalock-Taussig shunt $(\mathrm{n}=1)$ in some cases made it difficult to ascertain for pulmonary vessel opacification and assign a Spicer score. However, no collateral vessels were identified in the patients with patent ductus arteriosus, and grade 1 collaterals were identified by 1 reviewer in the patient with a modified Blalock-Taussig shunt.

\section{Angiogenic Factors, Protein, and Cells}

We measured arterial and venous plasma levels of the following hypoxia-inducible factors, which are known to be involved in sprouting angiogenesis: VEGF-A, C, D, bFGF, SDF-1a, and sFlt-1 (Figure 2). ${ }^{17}$ Compared with controls, patients with SV-APC had significantly higher arterial VEGF (a.k.a. VEGF-A) (58.7 pg/mL, 44-78.2 pg/mL vs
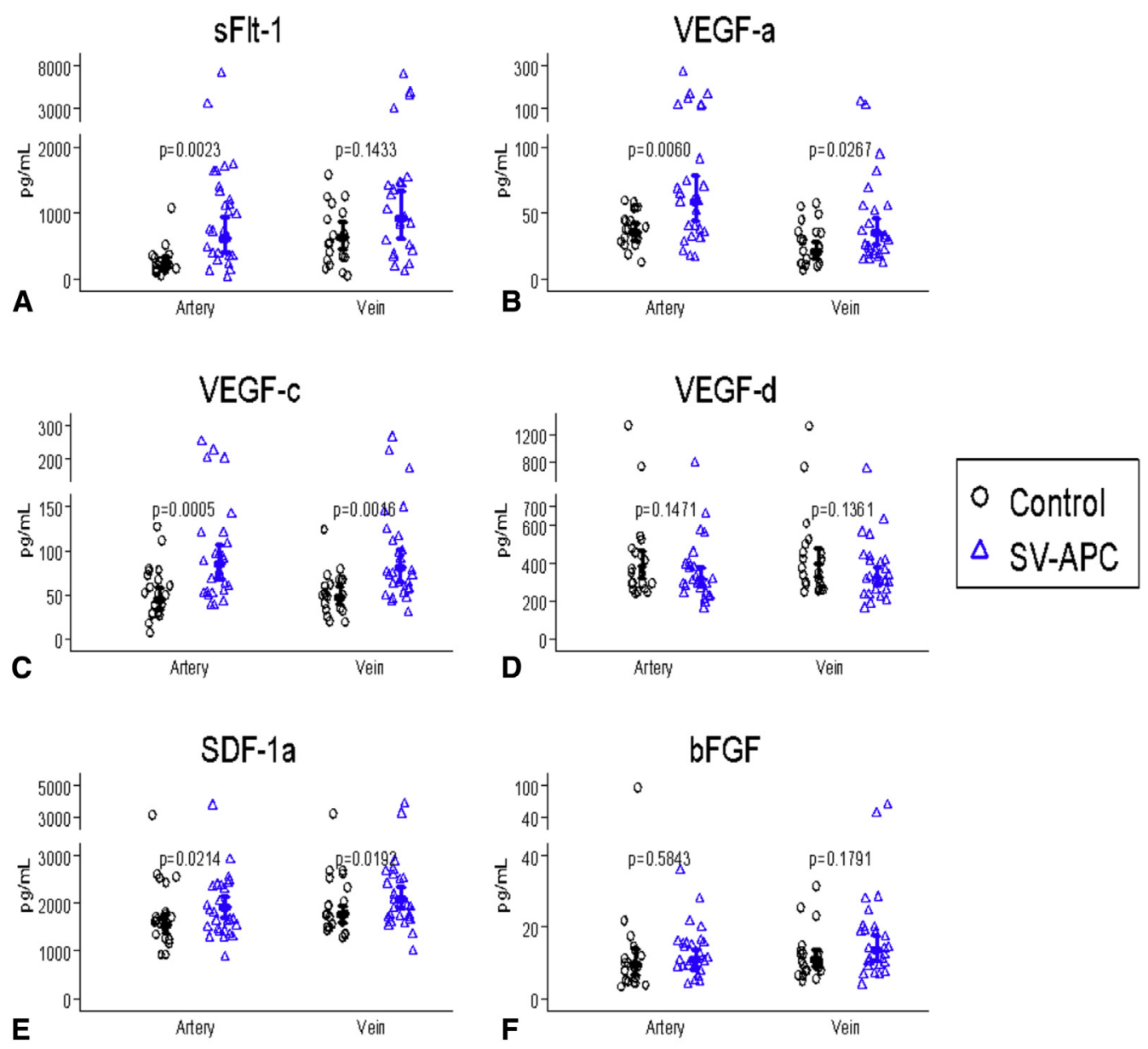

FIGURE 2. Levels of plasma angiogenic factors sampled from the femoral artery and vein in patients with SV-APC (blue triangles) and control patients (circles). All data are represented as mean values and 95\% CIs with individual data points overlaid. A, Plasma sFlt-1 levels. B, Plasma VEGF-A levels. C, Plasma VEGF-C levels. D, Plasma VEGF-D levels. E, Plasma SDF-1a levels. F, Plasma bFGF levels. sFlt-1, Soluble fms-like tyrosine kinase 1; $V E G F$, vascular endothelial growth factor; $S V-A P C$, single ventricle with aortopulmonary collateral; $S D F-1 a$, stromal-derived factor 1 alpha; $b F G F$, basic fibroblastic growth factor. 
$35.3 \mathrm{pg} / \mathrm{mL}, 29.3-42.6 ; P<.01)$ and venous VEGF $(34.8$ $\mathrm{pg} / \mathrm{mL}, 26.3-46 \mathrm{pg} / \mathrm{mL}$ vs $21 \mathrm{pg} / \mathrm{mL}, 15.6-28.2 ; P<.03)$. Patients with SV-APC also had significantly increased arterial SDF-1a (1901.6 pg/mL, 1694.5-2134 pg/mL vs $1542.6 \mathrm{pg} / \mathrm{mL}, 1355-1756.2 ; P<.03)$ and venous SDF-1a (2092.8 pg/mL, $1880.9-2328.6 \mathrm{pg} / \mathrm{mL}$ vs $1752.9 \mathrm{pg} / \mathrm{mL}$, 1584.9-1938.8; $P<.02$ ). Finally, patients with SV-APC had increased arterial sFlt-1 (612.3 pg/mL, 399-939.6 $\mathrm{pg} / \mathrm{mL}$ vs $243.1 \mathrm{pg} / \mathrm{mL}, 173.7-340.1 ; P<.003)$. Although most VEGF-C measurements fell below the assay's lower limit of quantification $(146 \mathrm{pg} / \mathrm{mL})$, patients with SV-APC had significantly increased arterial $(84.6 \mathrm{pg} / \mathrm{mL}, 67.4-106.1$ $\mathrm{pg} / \mathrm{mL}$ vs $44.3 \mathrm{pg} / \mathrm{mL}, 33.8-58.1 ; P<.001)$ and venous (81.2 pg/mL, 65.2-101.1 pg/mL vs $47.5 \mathrm{pg} / \mathrm{mL}, 38.4-58.9$; $P<.002)$ levels. No other factors were significantly different between groups. In all instances, there was wide variability in SV-APC patient factor levels and considerable overlap with controls. Total protein levels were similar in both groups (SV-APC: $5.3 \mathrm{~g} / \mathrm{dL}, 5.2-5.5$; control: $5.3 \mathrm{~g} / \mathrm{dL}, 5.1-5.4$, $P=.49$ ). Although hemoglobin and hematocrit were significantly elevated in the SV-APC group (data not shown, $P<.001)$, leukocytes $(P=.44)$ and platelet $(P=.58)$ counts were not different.

\section{Plasma-Induced Angiogenic Sprouting Activity}

We evaluated the effects of arterial and venous plasma on inducing HUVEC sprout formation in our 3-dimensional culture system (Figure 3). Initially, we validated our assay by demonstrating robust cell sprouting in the presence of endothelial cell growth media that contains several
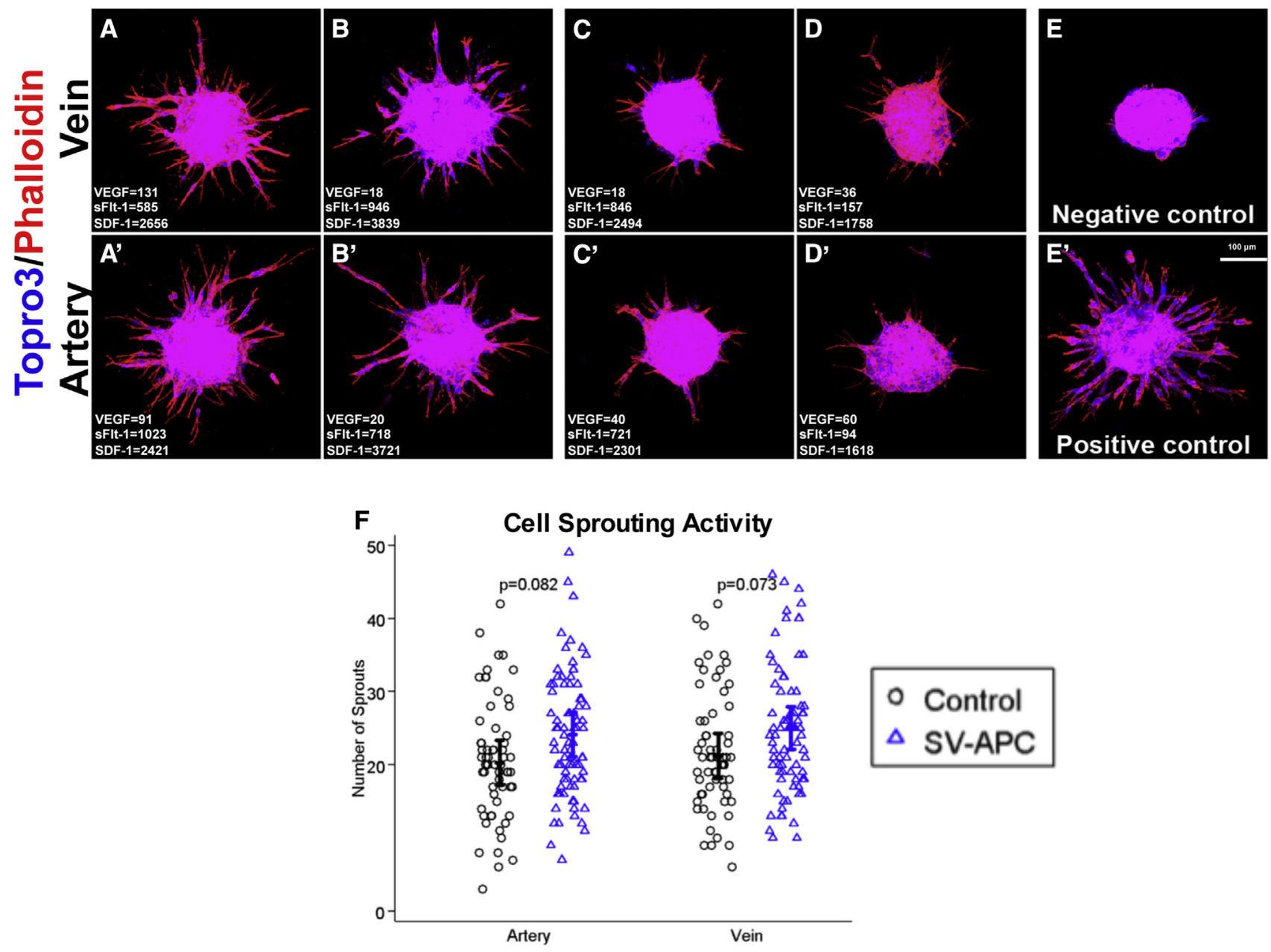

FIGURE 3. Plasma of patients with $S V$ physiology displays a wide range of angiogenic activity in vitro. Confocal microscopy images (20 $\times$ magnification) of fluorescently stained endothelial cell macrospheres incubated with venous (A-E) and arterial ( $\left.\mathrm{A}^{\prime}-\mathrm{E}^{\prime}\right)$ plasma from 3 patients with SV-APC (A-C) and 1 acyanotic biventricular control patient (D). Patients A and B have greater cell sprouting activity and increased plasma VEGF-A or SDF-1a levels (pg/mL) compared with patients $\mathrm{C}$ and D. Positive and negative controls with a $100 \mu \mathrm{m}$ scale bar are shown for reference (E). Overall, there was so significant difference in sprouting activity between patients with SV-APC (blue triangles) and control patients (circles) (F). Data are represented as mean values and $95 \%$ CIs with individual data points overlaid. VEGF, Vascular endothelial growth factor; $s F l t-1$, soluble fms-like tyrosine kinase $1 ; S D F-1 a$, stromal-derived factor 1 alpha; $S V-A P C$, single ventricle with aortopulmonary collateral. 
angiogenic factors (eg, VEGF and bFGF) and little to no sprouting with factor-free and serum-free basal media (Figure 3,E and $E^{\prime}$ ). We also found that plasma from a healthy volunteer can stimulate sprout formation in a dose-dependent manner (data not shown).

Although a few patients with SV-APC exhibited high sprouting activity (Figure $3, A, A^{\prime}, B$, and $B^{\prime}$ ) compared with control patients (Figure $3, D$ and $D^{\prime}$ ), other patients with SV-APC did not (Figure 3, $C$ and $C^{\prime}$ ). There was a wide variability in sprouting activity in the SV-APC group, which overlapped considerably with control patients. Although there was a trend toward an increased mean number of sprouts formed in the SV-APC group, this was not statistically significant in both artery $(P=.082)$ and vein $(P=.073)$ (Figure $3, F)$.

\section{Correlation of Plasma Angiogenic Factors, Cell Sprouting, and Disease Severity}

Because of their involvement in other pathologic conditions, we explored whether plasma VEGF-A, SDF$1 \mathrm{a}$, and sFlt-1 levels were associated with cell sprouting activity in vitro or clinical APC severity as assessed by the Spicer score. In the entire study cohort, venous cell sprouting was significantly positively correlated with VEGF-A $(P<.01)$ (Figure 4). A similar positive correlation was observed with venous SDF-1a, which was not statistically significant $(P=.06)$. No associations were found with arterial data. Regardless of sampling site, APC severity scores did not correlate with cell sprouting activity and factor levels (Figure 1, B-E).

\section{DISCUSSION}

To our knowledge, this is the first study to correlate angiographic assessment of APC burden with plasma hypoxia inducible factor measurements and in vitro

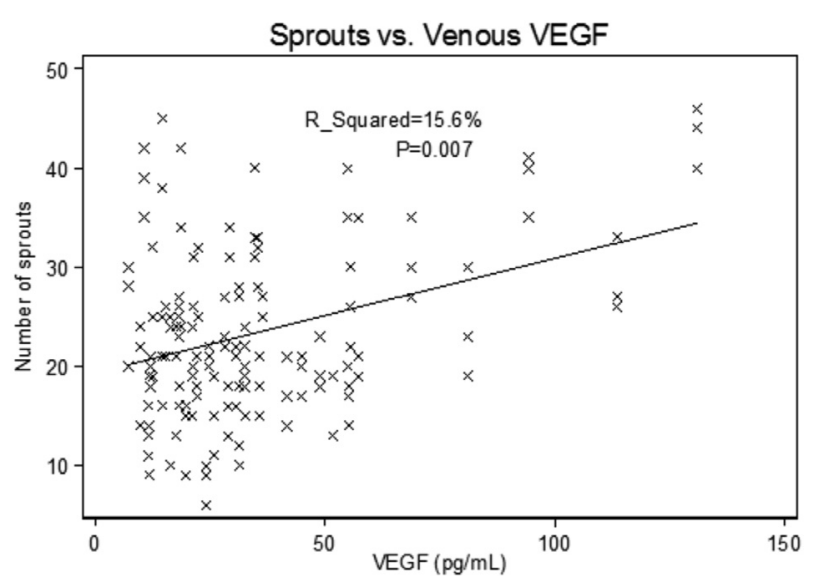

FIGURE 4. Venous plasma VEGF-A was positively correlated with cell sprouting activity in all 48 patients. $V E G F$, Vascular endothelial growth factor. angiogenic activity of SV patient plasma. We hypothesized that these patients would have significantly increased plasma levels of several hypoxia inducible factors, which would stimulate increased cell sprouting in vitro. Surprisingly, we found a wide range of in vitro angiogenic activity and factor levels that overlap considerably with control patients and correlate poorly with APC severity, suggesting a complex mechanism of angiogenesis (Figures 1,2, and 3). We have demonstrated for the first time that patients with SV physiology have significantly elevated arterial and venous SDF-1a, and arterial sFlt-1, both of which may participate in APC formation.

We considered confounding variables that could have affected our results. For instance, age was significantly different between SV-APC and control groups. A relationship between age and VEGF has been observed in early infancy; however, this does not seem to be an issue beyond age 3 months, which is when all study patients were sampled. $^{8,12}$ We also adjusted for age in all of our analyses to minimize its impact as a confounding variable. In addition, the range of VEGF levels we measured was somewhat lower than the range reported in most previous studies. ${ }^{8,10,27}$ Whereas other studies analyzed serum, we analyzed plasma in which VEGF values are often lower; plasma measurements are considered more physiologically representative than serum, and our results resemble those of a previous study that also analyzed plasma. ${ }^{12,28} \mathrm{We}$ also showed that total protein in both patient groups are similar and likely not confounding factor level measurements. Finally, it is also possible that our HUVEC assay may not adequately recapitulate APC pathogenesis, and alternative mechanisms such as enlargement of existing vascular connections (eg, bronchial arteries) may exist. However, the positive correlation we observed with plasma VEGF and cell sprouting supports the validity of our assay in assessing plasma angiogenic activity.

It is possible that factors other than the ones we studied are involved in APC pathogenesis. If the right factors were selected, however, a difference between patients with SV-APC and control patients could still have been masked given the time course during which angiogenesis occurs. Specifically, our data suggest a mechanism where upregulation and elevation of plasma angiogenic factors to create APCs may be a transient rather than a persistent state (Figure 1,F). Physiologically, the time at which patients were enrolled and sampled is arbitrary and may have occurred during an angiogenically active or dormant phase. The significant relationship we observed between cell sprouting and VEGF suggest that we sampled patients from both phases (Figure 4). In a dormant state, previously formed collaterals may be visualized angiographically, whereas plasma angiogenic activity (eg, factor levels and sprouts formed) is low. Likewise, in an angiogenically 
active state, there may be a time lag from when angiogenic activity peaks to when collateral vessels are visible angiographically. This is supported by the poor correlation of APC Spicer score with cell sprouting and plasma factor levels (Figure 1, $B-E$ ).

Although all the factors we assayed are regulated by the transcription factor hypoxia-inducible factor 1-alpha, a relationship between systemic hypoxemia and factor levels has been inconsistently documented. ${ }^{11,12,27}$ Our proposed mechanism where factors downregulate back to normal levels, because of a stimulus like chronic hypoxia, could explain these conflicting results. This phenomenon has been demonstrated in patients with chronic limb ischemia. ${ }^{24}$ Our hypothetical mechanism also could explain why some investigators found a relationship between VEGF and APCs, whereas others did not. ${ }^{10-12}$ Although we have graphically depicted only 1 peak (Figure $1, E$ ), the true pathophysiology may better resemble a sine wave with multiple peaks and valleys. Conflicting observations regarding the relationship of patient age (ie, duration of hypoxemia) to APC severity support a model where angiogenic processes may get turned on and off over time. ${ }^{29,30}$ If APC pathogenesis is cyclical as we propose, this has important implications for diagnosis and treatment of patients who have previously formed APCs but are now angiogenically dormant. Blood-based angiogenesis assays or biomarker tests could have poor sensitivity in these patients limiting their utility. Likewise, although antiangiogenic agents such as bevacizumab could have therapeutic potential by blocking VEGF signaling, they may not affect APCs that were previously formed.

The source of plasma angiogenic factors that form APCs is still unclear. Prior studies found no significant differences in respective factor levels when sampling blood in patients with APCs from the superior and inferior vena cavae, as well as from a systemic artery. ${ }^{8,10}$ In the present study, we were surprised to find such variation in factors such as VEGF and sFlt-1 levels between the femoral artery and the vein in both patient groups. Although identifying the mechanism for this variation is outside the scope of our present study, the trends we observed may help localize a source of these factors. From a practical standpoint, knowing the source will determine whether a reliable biomarker can be identified from venous or arterial blood.

Angiogenesis is a complex process requiring many steps and factors. Among the angiogenic factors previously studied in patients with APCs, only VEGF has emerged as a candidate pathogenic factor in some but not all studies. To our knowledge, we are the first to report significantly increased levels of arterial and venous VEGF together with arterial sFlt-1 (also known as "VEGF receptor 1"), as well as increased arterial and venous SDF-1a. VEGF primarily signals by binding to VEGF receptor 2 , whereas the role of sFlt-1 is less understood. Flt-1 exists in both membrane bound and soluble forms (sFlt-1) and is traditionally thought to exert antiangiogenic effects by trapping and preventing circulating VEGF from signaling via VEGF receptor $2 .{ }^{17}$ However, several studies support a potential proangiogenic role for sFlt-1. As endothelial cells sprout from a parent vessel to create branches, guidance cues are necessary to direct elongation so emerging sprouts will not curl back on themselves or collide with other sprouts. ${ }^{17}$ In embryonic murine stem cells, sFlt-1 knockout caused endothelial sprouts to emanate randomly without a direction; transgenic endogenous sFlt-1 expression rescued this phenotype by restoring a direction for elongation. ${ }^{20}$ Soluble Flt-1 also has been shown to promote interactions between endothelial and mural cells leading to vessel maturation and stability. ${ }^{22}$ Furthermore, elevated sFlt-1 levels have been measured in patients with other hypoxic and inflammatory diseases, such as pre-eclampsia and sepsis. ${ }^{21,23}$ Thus, although sFlt-1 may not itself stimulate sprout formation, our results coupled with previous findings suggest that it may help provide order, directionality, and stability during APC sprouting angiogenesis. SDF-1a (a.k.a. CXCL12) is a well-known hypoxia-inducible chemoattractant molecule whose role in vascular formation has been well documented in the gut, kidney, and retina. ${ }^{31-33}$ Its proangiogenic effect involves recruiting and retaining proangiogenic bone marrow-derived cells to areas of active angiogenesis. ${ }^{17}$ With the use of homozygous knockout of SDF-1a or its receptor CXCR4, we previously demonstrated that this signaling axis works together with VEGF to promote nerve-vessel alignment and arteriogenesis in murine embryonic limb skin. ${ }^{18}$ Increased expression of VEGF and SDF-1a also has been demonstrated in ischemic versus nonischemic muscle in patients undergoing limb amputation for peripheral vascular disease. ${ }^{24}$ Finally, increased VEGF, SDF-1a, and circulating endothelial progenitor cells have been documented in cyanotic children with tetralogy of Fallot. ${ }^{19}$ These results together with our findings suggest that sFlt-1 and SDF-1a may be involved in APC formation.

\section{Study Limitations}

A major limitation of our study is that invasive contrast angiography can have poor sensitivity in detecting APC severity because of variations in technique..$^{3,5,6,13}$ The Spicer scoring system, although practical, is an ordinal and subjective scale that does not represent the full spectrum of disease. ${ }^{13}$ It also does not assess for other pathologic angiogenesis unrelated to APCs, such as pulmonary arteriovenous malformations (seen in patients with SV physiology) or physiologic angiogenesis in controls. The presence of pulmonary arteriovenous malformations could have contributed to the poor correlation of APC Spicer score with cell sprouting and plasma factor 
levels. Although all patients would have ideally undergone phase-contrast MRI to quantify APC flow, this is not a routine clinical practice at our institution. A second limitation arises from inherent variability between groups with respect to age and within groups in terms of diagnosis and anatomy, which increases susceptibility to confounding variables. These various heterogeneities may weaken the strength of the various differences and correlations (or lack thereof) we reported. These limitations arise in part from our inability to perform invasive blood sampling and aortography in age-matched healthy volunteers. Further, all study patients would have ideally been sampled at multiple time points, which is not ethically and practically feasible. Without these data, we cannot definitively support our proposed time course mechanism of APC formation. Finally, despite our efforts to standardize all cell sprouting experiments, we acknowledge that slight differences in conditions from run to run could have confounded our sprouting data. Because we are the first to use the cell sprouting assay with human plasma, the expected magnitude of difference in sprouting activity is not known and our study may have been underpowered to detect a difference.

\section{CONCLUSIONS}

Our current understanding of APC vessel pathogenesis is limited. The present study has demonstrated that patients with SV physiology exhibit a wide range of plasma angiogenic activity that correlates poorly with disease severity, which may be explained by a time-dependent phenomenon. If true, this would limit the utility of bloodbased methods of diagnosing APCs. Stromal-derived factor and soluble flt-1, a regulatory VEGF receptor, are elevated in patients with SV physiology, and their roles in APC pathogenesis require further investigation.

\section{Conflict of Interest Statement}

Authors have nothing to disclose with regard to commercial support.

The authors thank the many individuals who supported and contributed to this project: CNHS Division of Hematology: Naomi Luban and Lori Luchtman-Jones provided project funding and mentorship. CNHS Division of Cardiology: Catherine Connors, Ileen Cronin, Charles Fleming, Manuel Mendez III, and Beth Thompson performed blood collections. Lowell Frank and E. Anne Greene provided scholarly guidance. CNHS Clinical Research Center: Pablo Cure and Amanda Kasper provided additional sources of intramural funding. Jayna Bryant, Vonterris Hagan-Temple, Ianka Laidlow, Marlene Lee, Brenda Martin, Vera Okoye, and Lisa Pickett-Evans performed blood processing and plasma isolation. National Heart, Lung, and Blood Institute: Faye Baldrey and Rachel Reed provided administrative assistance. Christian Combs, Julia Doveikis, Krista Gill, Christopher Hourigan, Nehal Mehta, Balaji Natarajan, Martin Playford, Shawn
Rose, and Mikhael Wallowitz (Mesoscale Discovery) provided technical expertise, equipment, and consultations. The authors also thank the other members of Laboratory of Stem Cell and Neuro-Vascular Biology for technical help and thoughtful discussion.

\section{References}

1. Khairy P, Poirier N, Mercier LA. Univentricular heart. Circulation. 2007;115: 800-12.

2. Hoffman JI, Kaplan S. The incidence of congenital heart disease. J Am Coll Cardiol. 2002;39:1890-900.

3. Bradley SM, McCall MM, Sistino JJ, Radtke WA. Aortopulmonary collateral flow in the Fontan patient: does it matter? Ann Thorac Surg. 2001; 72:408-15.

4. Kanter KR, Vincent RN. Management of aortopulmonary collateral arteries in Fontan patients: occlusion improves clinical outcome. Semin Thorac Cardiovasc Surg Pediatr Card Surg Annu. 2002;5:48-54.

5. McElhinney DB, Reddy VM, Tworetzky W, Petrossian E, Hanley FL, Moore P. Incidence and implications of systemic to pulmonary collaterals after bidirectional cavopulmonary anastomosis. Ann Thorac Surg. 2000;69:1222-8.

6. Triedman JK, Bridges ND, Mayer JE Jr, Lock JE. Prevalence and risk factors for aortopulmonary collateral vessels after Fontan and bidirectional Glenn procedures. J Am Coll Cardiol. 1993;22:207-15.

7. Prakash A, Satiroglu E, Porras D, McElhinney DB, Keane JF, Lock JE, et al. Risk factors for profuse systemic-to-pulmonary artery collateral burden in hypoplastic left heart syndrome. Am J Cardiol. 2013;112:400-4.

8. Starnes SL, Duncan BW, Kneebone JM, Rosenthal GL, Jones TK, Grifka RG, et al. Vascular endothelial growth factor and basic fibroblast growth factor in children with cyanotic congenital heart disease. J Thorac Cardiovasc Surg. 2000;119:534-9.

9. Hamada H, Ebata R, Higashi K, Tateno S, Niwa K, Honda T, et al. Serum vascular endothelial growth factor in cyanotic congenital heart disease functionally contributes to endothelial cell kinetics in vitro. Int J Cardiol. 2007; 120:66-71.

10. Mori Y, Shoji M, Nakanishi T, Fujii T, Nakazawa M. Elevated vascular endothelial growth factor levels are associated with aortopulmonary collateral vessels in patients before and after the Fontan procedure. Am Heart J. 2007; 153:987-94.

11. Ootaki Y, Yamaguchi M, Yoshimura N, Oka S, Yoshida M, Hasegawa T. Vascular endothelial growth factor in children with congenital heart disease. Ann Thorac Surg. 2003;75:1523-6.

12. Himeno W, Akagi T, Furui J, Maeno Y, Ishii M, Kosai $K$, et al. Increased angiogenic growth factor in cyanotic congenital heart disease. Pediatr Cardiol. 2003;24:127-32.

13. Spicer RL, Uzark KC, Moore JW, Mainwaring RD, Lamberti JJ. Aortopulmonary collateral vessels and prolonged pleural effusions after modified Fontan procedures. Am Heart J. 1996;131:1164-8.

14. Wernovsky G, Bridges ND, Mandell VS, Castaneda AR, Perry SB. Enlarged bronchial arteries after early repair of transposition of the great arteries. J Am Coll Cardiol. 1993;21:465-70.

15. Grosse-Wortmann L, Al-Otay A, Yoo SJ. Aortopulmonary collaterals after bidirectional cavopulmonary connection or Fontan completion: quantification with MRI. Circ Cardiovasc Imaging. 2009;2:219-25.

16. Whitehead KK, Gillespie MJ, Harris MA, Fogel MA, Rome JJ. Noninvasive quantification of systemic-to-pulmonary collateral flow: a major source of inefficiency in patients with superior cavopulmonary connections. Circ Cardiovasc Imaging. 2009;2:405-11.

17. Carmeliet P, Jain RK. Molecular mechanisms and clinical applications of angiogenesis. Nature. 2011;473:298-307.

18. Li W, Kohara H, Uchida Y, James JM, Soneji K, Cronshaw DG, et al. Peripheral nerve-derived CXCL12 and VEGF-A regulate the patterning of arterial vessel branching in developing limb skin. Dev Cell. 2013;24:359-71.

19. Liu ZL, Wu ZS, Hu JG, Yang YF, Chen Y, Gao H, et al. Correlation of serum levels of VEGF and SDF-1 with the number and function of circulating EPCs in children with cyanotic congenital heart disease. Zhongguo Dang Dai Er Ke Za Zhi. 2009;11:267-72.

20. Chappell JC, Taylor SM, Ferrara N, Bautch VL. Local guidance of emerging vessel sprouts requires soluble flt-1. Dev Cell. 2009;17:377-86.

21. Nevo O, Soleymanlou N, Wu Y, Xu J, Kingdom J, Many A, et al. Increased expression of sFlt-1 in in vivo and in vitro models of human placental hypoxia 
is mediated by HIF-1. Am J Physiol Regul Integr Comp Physiol. 2006;291: R1085-93.

22. Lorquet S, Berndt S, Blacher S, Gengoux E, Peulen O, Maquoi E, et al. Soluble forms of VEGF receptor-1 and -2 promote vascular maturation via mural cell recruitment. FASEB J. 2010;24:3782-95.

23. Ebihara I, Hirayama K, Kaneko S, Nagai M, Ogawa Y, Fujita S, et al. Vascular endothelial growth factor and soluble fms-like tyrosine kinase-1 in septic shock patients treated with direct hemoperfusion with a polymyxin B-immobilized fiber column. Ther Apher Dial. 2008;12:285-91.

24. van Weel V, Seghers L, de Vries MR, Kuiper EJ, Schlingemann RO, Bajema IM, et al. Expression of vascular endothelial growth factor, stromal cell-derived factor-1, and CXCR4 in human limb muscle with acute and chronic ischemia. Arterioscler Thromb Vasc Biol. 2007;27:1426-32.

25. Rossi RN, Hislop A, Anderson RH, Martins FM, Cook AC. Systemic-topulmonary blood supply in tetralogy of Fallot with pulmonary atresia. Cardiol Young. 2002;12:373-88.

26. Smith AO, Bowers SL, Stratman AN, Davis GE. Hematopoietic stem cell cytokines and fibroblast growth factor-2 stimulate human endothelial cell-pericyte tube co-assembly in 3D fibrin matrices under serum-free defined conditions. PLoS One. 2013;8:e85147.

27. Suda K, Matsumura M, Miyanish S, Uehara K, Sugita T, Matsumoto M. Increased vascular endothelial growth factor in patients with cyanotic congenital heart diseases may not be normalized after a Fontan type operation. Ann Thorac Surg. 2004;78:942-7.

28. Jelkmann W. Pitfalls in the measurement of circulating vascular endothelial growth factor. Clin Chem. 2001;47:617-23.

29. Kanter KR, Vincent RN, Raviele AA. Importance of acquired systemic-topulmonary collaterals in the Fontan operation. Ann Thorac Surg. 1999;68: 969-75.

30. Ichikawa H, Yagihara T, Kishimoto H, Isobe F, Yamamoto F, Nishigaki K, et al Extent of aortopulmonary collateral blood flow as a risk factor for Fontan operations. Ann Thorac Surg. 1995;59:433-7.

31. Ara T, Tokoyoda K, Okamoto R, Koni PA, Nagasawa T. The role of CXCL12 in the organ-specific process of artery formation. Blood. 2005; 105:3155-61.

32. Strasser GA, Kaminker JS, Tessier-Lavigne M. Microarray analysis of retina endothelial tip cells identifies CXCR4 as a mediator of tip cell morphology and branching. Blood. 2010;115:5102-10.

33. Takabatake Y, Sugiyama T, Kohara H, Matsusaka T, Kurihara H, Koni PA, et al The CXCL12 (SDF-1)/CXCR4 axis is essential for the development of renal vasculature. J Am Soc Nephrol. 2009;20:1714-23.

Key Words: single ventricle, aortopulmonary collateral, congenital heart disease

\title{
EDITORIAL COMMENTARY
}

\section{Aortopulmonary collaterals: Angiogenesis, or a whole lot more?}

\author{
Winfield J. Wells, MD, and S. Ram Kumar, MD, PhD
}

\footnotetext{
From the Division of Cardiac Surgery, Department of Surgery, University of Southern California; and Heart Institute, Children's Hospital of Los Angeles, Los Angeles, Calif.

Disclosures: Authors have nothing to disclose with regard to commercial support.

Received for publication Oct 21, 2015; accepted for publication Oct 22, 2015; available ahead of print Nov 24, 2015.

Address for reprints: Winfield J. Wells, MD, Children's Hospital of Los Angeles, 4650 Sunset Blvd, Mailstop \#66,

Los Angeles, CA 90033 (E-mail: wwells@ chla.usc.edu).

J Thorac Cardiovasc Surg 2016;151:1135-6

$0022-5223 / \$ 36.00$

Copyright (c) 2016 by The American Association for Thoracic Surgery

http://dx.doi.org/10.1016/j.jtcvs.2015.10.086
}

Favorable pulmonary vascular dynamics is the key element of successful Fontan circulation. Unfortunately, alterations in the pulmonary vascular tree are not uncommon in children who have complex congenital heart disease. Aortopulmonary collaterals (APC), which divert oxygenated blood from the systemic circulation back to the lungs, are the most commonly acquired pulmonary vascular abnormalities. Several investigators have attempted to identify the physiologic stimuli for the formation and progression of APC. As the field of molecular biology has matured, additional efforts have been made to define the molecular basis for the formation of APC.

Along those lines, this article by Sandeep and colleagues ${ }^{1}$ seeks to correlate the serum level of angiogenic factors with angiogenic activity in vitro, and APC formation in vivo.

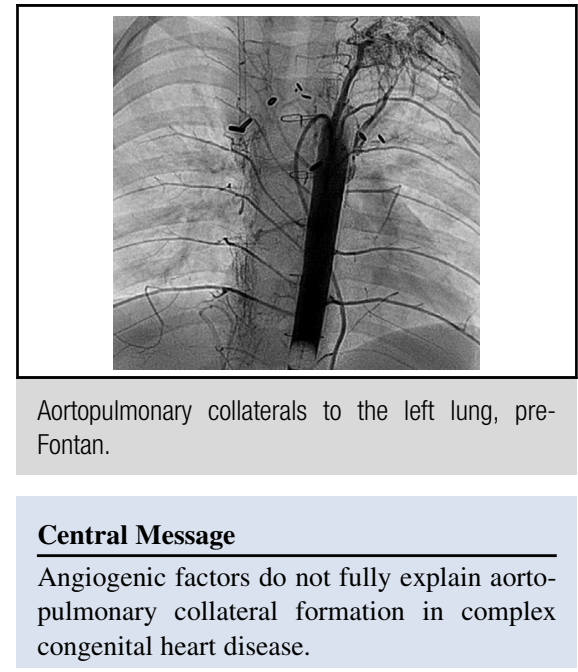

See Article page 1126.

Although factor concentration in the serum differed significantly between the 2 groups of patients studied, it was poorly correlated with the extent of APC. Despite this negative result, the authors should be congratulated for pursuing what many investigators would have regarded as too simplistic a hypothesis. 


\section{APPENDIX}

\section{Cell sprouting assay}

To standardize all experiments as much as possible, all HUVECs were derived from a commercially available single vial of pooled donor primary cells (Lonza Ltd, Basel, Switzerland). Cell colonies were expanded and grown from this vial and passaged 3 times over 9 days in $55 \mathrm{~cm}^{2}$ culture dishes (Corning Life Sciences, Tewksbury, Mass) before being stocked into liquid nitrogen. Growth never exceeded $70 \%$ to $80 \%$ confluency. For each experiment, cells were plated onto $55 \mathrm{~cm}^{2}$ culture dishes with endothelial cell growth media (Lonza Ltd, Basel, Switzerland). After 3 days, cells were washed, trypsinized, counted, resuspended in endothelial growth media, and cultured in a 96-well clear round bottom ultra-low attachment microplate (Corning Life Sciences) for 27 to 28 hours to produce macrospheres comprising approximately 2000 cells. Macrospheres were gently embedded into a 3-dimensional collagen matrix (Nitta Gelatin, Osaka, Japan) and applied to $9.6 \mathrm{~cm}^{2}$ glass-bottom culture dishes
(In Vitro Scientific, Sunnyvale, Calif). After incubation at $37^{\circ} \mathrm{C}$ with $5 \%$ carbon dioxide for 30 to 40 minutes, $1 \mu \mathrm{L}$ of patient plasma diluted in $2 \mathrm{~mL}$ of serum-free and factor-free endothelial cell basal media (Lonza Ltd) was applied to each collagen-embedded HUVEC macrosphere. All plasma samples were assayed in triplicate. After incubating for 20 to 21 hours at $37^{\circ} \mathrm{C}$ with $5 \%$ carbon dioxide, cells were fixed with $4 \%$ paraformaldehyde, stained with fluorescent dyes (To-pro-3, pan-nuclear marker and Phalloidin, actin cytoskeleton marker), visualized using a Leica TCS SP5 confocal microscope (Leica, Wetzlar, Germany), and the number of induced sprouts was manually counted and averaged. To minimize run to run variability, patients with SV-APC and control patients were run in the same experiments as much as possible. HUVEC macrospheres were tested in every experimental run with angiogenically inert serum-free and factor-free basal media (negative control) and serum and growth factor containing endothelial cell growth media (positive control) (Figure 3,E and $E^{\prime}$ ). 
TABLE E1. Individual patient demographic data

\begin{tabular}{|c|c|c|c|c|}
\hline Individual anatomic diagnoses & Age (mo) & SpO2 $(\%)$ & Circulation & APC grade (score 1/score 2) \\
\hline \multicolumn{5}{|l|}{ Control group } \\
\hline Severe aortic stenosis & 3 & 99 & $2 \mathrm{~V}$ & $2 / 1$ \\
\hline Subaortic membrane & 111 & 95 & $2 \mathrm{~V}$ & $0 / 0$ \\
\hline Aortic stenosis s/p Ross & 233 & 96 & $2 \mathrm{~V}$ & $0 / 0$ \\
\hline Aortic stenosis and insufficiency & 195 & 99 & $2 \mathrm{~V}$ & $0 / 0$ \\
\hline Repaired coarctation + subaortic membrane & 34 & 99 & $2 \mathrm{~V}$ & $1 / 1$ \\
\hline Repaired coarctation + LPA stenosis & 35 & 97 & $2 \mathrm{~V}$ & $0 / 0$ \\
\hline Repaired coarctation + supravalvar AS & 84 & 99 & $2 \mathrm{~V}$ & $0 / 0$ \\
\hline PDA & 76 & 99 & $2 \mathrm{~V}$ & $0 / 0$ \\
\hline PDA & 4 & 96 & $2 \mathrm{~V}$ & $0 / 0$ \\
\hline PDA & 53 & 99 & $2 \mathrm{~V}$ & $0 / 0$ \\
\hline DORV-mBTS, 9 y since OHT & 100 & 99 & $2 \mathrm{~V}$ & $0 / 0$ \\
\hline PA/IVS, 9 y since OHT & 106 & 98 & $2 \mathrm{~V}$ & $0 / 0$ \\
\hline Tricuspid atresia-Fontan, 6 y since OHT & 237 & 99 & $2 \mathrm{~V}$ & $0 / 0$ \\
\hline Dilated cardiomyopathy, 2 y since OHT & 190 & 99 & $2 \mathrm{~V}$ & $0 / 0$ \\
\hline Congenital CHB, 6 y since OHT & 202 & 97 & $2 \mathrm{~V}$ & $0 / 0$ \\
\hline Transitional AV canal, active coarctation & 4 & 98 & $2 \mathrm{~V}$ & $0 / 0$ \\
\hline Transitional AV canal, subaortic membrane & 28 & 98 & $2 \mathrm{~V}$ & $0 / 0$ \\
\hline Mitral stenosis and insufficiency & 117 & 93 & $2 \mathrm{~V}$ & $0 / 0$ \\
\hline D-TGA s/p arterial switch & 11 & 97 & $2 \mathrm{~V}$ & $3 / 1$ \\
\hline Repaired truncus arteriosus & 39 & 99 & $2 \mathrm{~V}$ & $0 / 0$ \\
\hline Repaired aortic atresia with VSD & 102 & 97 & $2 \mathrm{~V}$ & $0 / 0$ \\
\hline \multicolumn{5}{|l|}{ SV-APC group } \\
\hline DORV, AV canal, heterotaxy & 53 & 86 & Fontan & $3 / 3$ \\
\hline DORV (MS, AS) & 58 & 87 & Glenn & $3 / 3$ \\
\hline DORV, severe pulmonary stenosis & 118 & 90 & Fontan & $1 / 1$ \\
\hline DORV $[S, D, D]$ & 22 & 84 & Glenn & $4 / 4$ \\
\hline DORV $[S, L, L]$, AV canal, heterotaxy & 23 & 79 & Glenn & $3 / 3$ \\
\hline DORV, pulmonary atresia & 35 & 90 & Glenn & $3 / 3$ \\
\hline DORV $[S, D, D]$, mitral atresia & 43 & 82 & Fontan & $3 / 3$ \\
\hline HLHS [MS, AS] & 81 & 87 & Hemi-Fontan & $3 / 3$ \\
\hline HLHS [MS, AA] & 30 & 86 & Glenn & $3 / 3$ \\
\hline HLHS [MS, AS] & 20 & 92 & Glenn & $3 / 4$ \\
\hline HLHS [MA, AA] & 58 & 87 & Fontan & $3 / 3$ \\
\hline HLHS [MA, AA] & 21 & 88 & Glenn & $3 / 3$ \\
\hline HLHS [MA, AA] & 5 & 80 & Norwood-Sano & $3 / 3$ \\
\hline HLHS [MA, AA] & 42 & 85 & Glenn & $3 / 3$ \\
\hline DILV [S,D,D $]$ & 23 & 80 & Glenn & $2 / 2$ \\
\hline DILV $[S, L, L]$ & 50 & 78 & Glenn & $3 / 1$ \\
\hline DILV $[S, L, L]$, pulmonary atresia & 19 & 89 & Glenn & $3 / 3$ \\
\hline DILV $[S, L, L]$ & 183 & 77 & Glenn & $3 / 3$ \\
\hline DILV [S,D,D], tricuspid atresia & 76 & 83 & Glenn & $3 / 3$ \\
\hline Tricuspid stenosis, D-TGA & 7 & 80 & Glenn & $3 / 3$ \\
\hline Tricuspid atresia, D-TGA & 4 & 76 & PA band & $3 / 3$ \\
\hline Tricuspid atresia, D-TGA & 3 & 79 & Norwood-mBTS & $1 / 0$ \\
\hline Tricuspid atresia, D-TGA & 28 & 87 & Glenn & $2 / 3$ \\
\hline PA/IVS & 17 & 83 & Glenn & $3 / 3$ \\
\hline PA/IVS & 21 & 79 & RF perforation of $\mathrm{PV}$ & $0 / 0$ \\
\hline $\mathrm{PA}, \mathrm{VSD}[\mathrm{S}, \mathrm{L}, \mathrm{X}]$ & 16 & 64 & Glenn & $3 / 3$ \\
\hline Unbalanced AV canal, heterotaxy & 82 & 86 & Glenn & $4 / 4$ \\
\hline
\end{tabular}

$S p O 2$, Oxygen saturation; $A P C$, aortopulmonary collateral; $2 V$, biventricular circulation; $L P A$, left pulmonary artery; $A S$, aortic stenosis; $P D A$, patent ductus arteriosus; $D O R V$, double outlet right ventricle; $m B T S$, modified Blalock-Taussig shunt; $O H T$, orthotopic heart transplant; $P A / I V S$, pulmonary atresia with intact ventricular septum; $C H B$, complete heart block; $A V$, atrioventricular; $D-T G A$, D-transposition of the great arteries; VSD, ventricular septal defect; $S V$ - $A P C$, single ventricle with aortopulmonary collateral; $M S$, mitral stenosis; $H L H S$, hypoplastic left heart syndrome; $A A$, aortic atresia; $M A$, mitral atresia; $D I L V$, double inlet left ventricle; $R F$, radiofrequency; $P V$, pulmonary valve. 